\title{
Profile of abortion seekers in Ghana and their decision-making processes
}

Philomena Nyarko

Population Council

Cletus Adohinzin

Saumya RamaRao

Population Council

Placide Tapsoba

Population Council

Ayorinde Ajayi

Population Council

Follow this and additional works at: https://knowledgecommons.popcouncil.org/departments_sbsr-rh

Part of the Demography, Population, and Ecology Commons, Family, Life Course, and Society

Commons, Gender and Sexuality Commons, International Public Health Commons, Maternal and Child

Health Commons, Medicine and Health Commons, and the Women's Health Commons

How does access to this work benefit you? Let us know!

\section{Recommended Citation}

Nyarko, Philomena, Cletus Adohinzin, Saumya RamaRao, Placide Tapsoba, and Ayorinde Ajayi. 2008.

"Profile of abortion seekers in Ghana and their decision-making processes." Accra: Population Council. 


\section{Profile OF AborTION SEEKERS IN GHANA AND THEIR DECISION-MAKING PROCESSES}

July 22, 2008

Philomena Nyarko ${ }^{1}$, Clétus Adohinzin ${ }^{2}$, Saumya RamaRao ${ }^{2}$, Placide Tapsoba $^{2}$ and Ayo Ajayi ${ }^{2}$ 


\section{EXECUTIVE SUMMARY}

In Ghana, it is widely believed that abortions are among the leading causes of maternal mortality. With a maternal mortality ratio of 540 per 100,000 live births (World Health Organization, 2006), efforts to improve maternal survival need to pay particular attention to unwanted pregnancy and abortion.

Since 1985, Ghanaian law allows abortion under specific circumstances. However, translation of the law into effective services has been limited. Since knowledge of the abortion law and its nuances is low in communities and there is sensitivity around abortions (both miscarriages and induced abortions), it is difficult to derive reliable community level estimates. Data gathered from facilities have limitations of incompleteness or inaccuracy; they also reflect the experiences of only those who have sought services and not other women. Lack of complete understanding of the abortion law makes providers unwilling to accurately classify the type of abortion (induced or spontaneous) and for women seeking care to admit to having induced an abortion.

The objective of this formative research was to provide a profile of beneficiaries, their needs for information and services, their decision-making processes to seek care, their knowledge of service points, and the quality of care they received. This information is expected to support service institutions in the design and implementation of client oriented services, especially those that aim to reduce maternal mortality.

The study is based on data collected from four health facilities located in the Greater Accra region where abortion services are offered: two public sector facilities and two private-sector facilities run by Marie Stopes International (MSI). Data were gathered through a review of patient case records, interviews with 12 providers, 145 observations of the interaction between providers and clients during consultation and 146 client exit interviews.

The results indicate that clients seeking abortion care in Ghana are mostly young adults. The age of the clients ranged from 14 to 41 years, with a mean of 25 years. The distribution by age group shows that the 20-24 year age group alone accounted for 41 percent of these clients while those aged between 14 and 19 years made up 18 percent. The clients who patronized abortion services were quite well educated. Two-fifths (37\%) had had middle or junior secondary school education while about a third had received senior secondary or higher level education. The data shows that quite a large number of the clients were engaged in sales (36\%) and service (23\%) occupations. Those in professional, technical, administrative and clerical occupations accounted for 10 percent while the unemployed and students made up 16 percent and 12 percent of the study sample respectively. Almost a quarter $(24 \%)$ of the clients lived with their husbands. The rest were mostly living with their sisters $(17.8 \%)$, mothers $(10.3 \%)$, both parents $(10.3 \%)$ or other relatives such as children, aunties, uncles and brothers-in-law (15.1\%).

In terms of maternity history and personal abortion experiences of clients, for 32 percent of the clients, this was the first pregnancy they were carrying. Among the 99 clients who had experienced an earlier pregnancy, 16 percent had no living children. 92.5 percent of the clients indicated that the current pregnancy was the result their willing. Only 7.5 percent of them had used a contraceptive method and the main methods adopted were the male condom (3.4\%) and the pill $(3.4 \%)$. The rest were either on the injectable $(1.4 \%)$ or had used a female condom $(0.7 \%)$ during the sexual interaction. 
In terms of the reasons why they had arrived at the decision not to have the child, $39.7 \%$ of client indicated that the pregnancy was either mistimed or unwanted either by themselves and $19.8 \%$ by their partners or other family members while $16.4 \%$ of them indicated that they needed to get rid of the pregnancy so that they could continue with their work or education. The majority of the client $(32 \%)$ indicated that they took the final decision themselves to come to the facility on that day while almost a quarter $(24.0 \%)$ mentioned that it was a joint decision between themselves and their partners. Thus, 55 percent of the clients had a say in the decision to seek services at the current facility.

From provider interviews it was found that the providers know some aspects of the Ghana standards and protocols on comprehensive abortion care but would like additional training for greater comprehension. Only 4 of the 12 staff who routinely provide abortion care reported that they knew the Ghana standards and protocols on comprehensive abortion care very well. Overall, the interpersonal communication between providers and clients was satisfactory at both the private and public health institutions. However, the providers from the private sector interacted better with the clients than the providers from the public sector. The research also highlights that that the information exchanged between providers and clients can be improved. Clients need to be informed of family planning options and services so that they can protect themselves from future unwanted pregnancies. 


\section{TABLe OF CONTENTS}

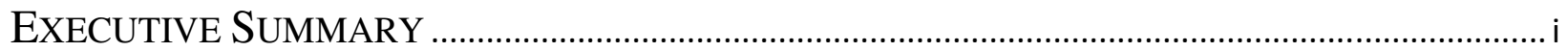

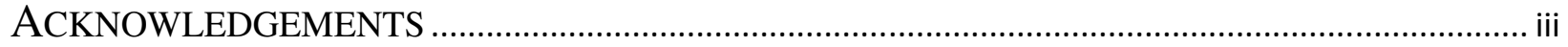

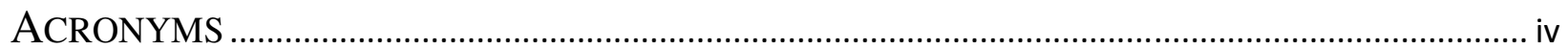

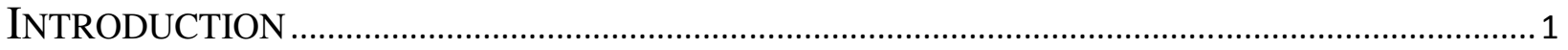

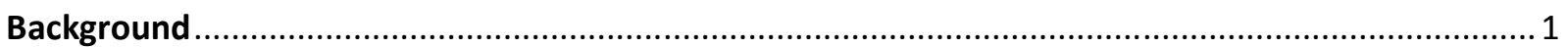

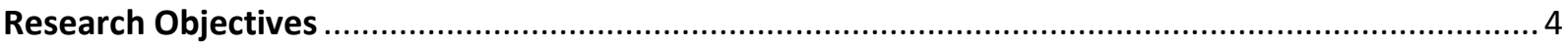

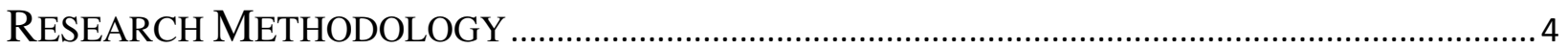

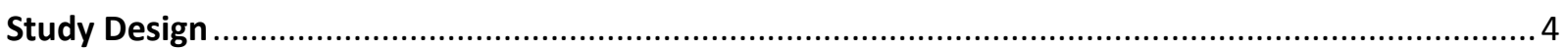

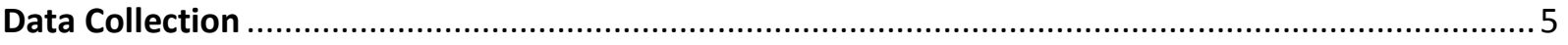

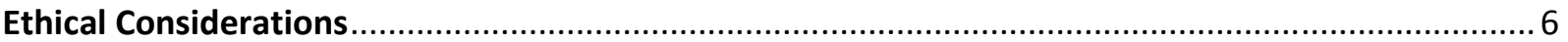

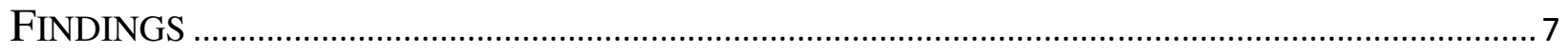

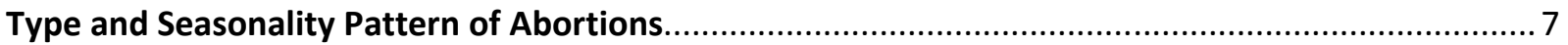

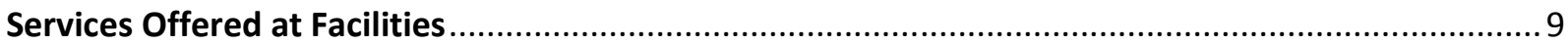

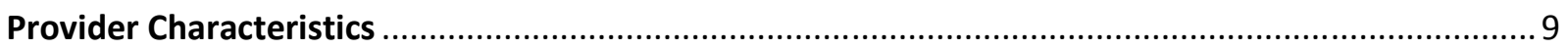

Pre-service Training and Knowledge of Service Guidelines and Abortion Law ............................... 10

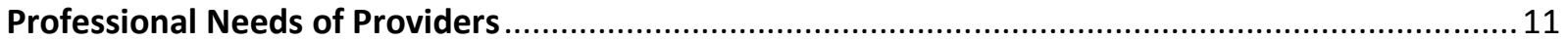

Quality of Care Given During Provider-client Interaction.................................................................. 12

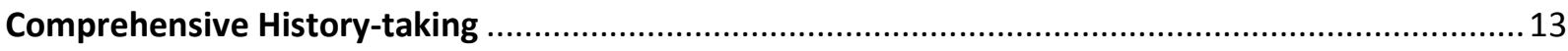

Quality of Examination Conducted During Consultation .............................................................. 14

Information and Counseling Given During Consultation .......................................................... 16

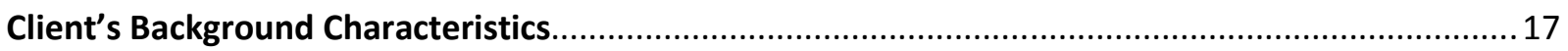

Maternity History and Personal Abortion Experiences of Clients ....................................................2 20

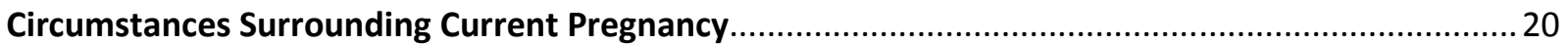

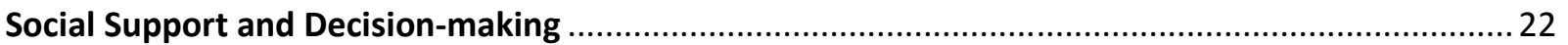

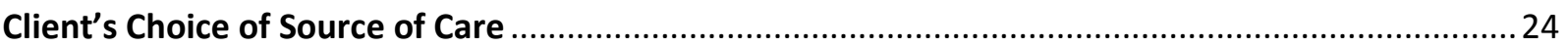

Clients' Assessment of Type, Cost and Quality of Services Received .............................................. 25

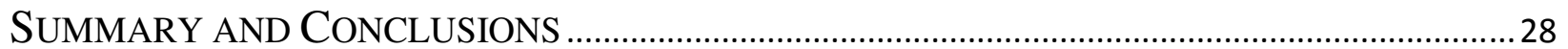

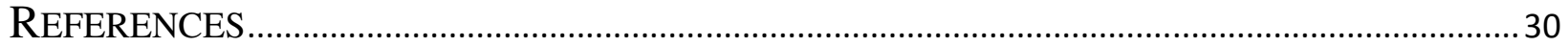




\section{ACKNOWLEDGEMENTS}

We would like to acknowledge the support of several organisations and individuals who contributed to this research, in particular staff of Marie Stopes International (MSI) and Ghana Health Service.

The contribution of all stakeholders interviewed before and during the data collection is deeply appreciated. Our special gratitude goes to the MSI staff, Mrs. Faustina Fynn-Nyame (Country Director) and Mr. Fred Gbagbo (Clinic Services Manager), to Dr Emmanuel Srofenyoh (Head of OB/GYN ward), Ridge Hospital and to Dr Dorcas Olga Anfu (Head of OB/GYN ward), La General Hospital for making this assessment possible. 


\section{ACRONYMS}

\begin{tabular}{|c|c|}
\hline AIDS & Acquired Immunodeficiency Syndrome \\
\hline CAC & Comprehensive Abortion Care \\
\hline $\mathrm{D} \& \mathrm{C}$ & Dilatation and Curettage \\
\hline $\mathrm{D} \& \mathrm{E}$ & Dilatation and Evacuation \\
\hline DHS & Demographic and Health Survey \\
\hline EVA & Electric Vacuum Aspiration \\
\hline GHS & Ghana Health Service \\
\hline HIV & Human Immune Virus \\
\hline IUD & Intrauterine Device \\
\hline MA & Medication Abortion \\
\hline MSI & Marie Stopes International \\
\hline MVA & Manual Vacuum Aspiration \\
\hline PAC & Post Abortion Care \\
\hline RH & Reproductive Health \\
\hline $\mathrm{R} 3 \mathrm{M}$ & Reducing Maternal Morbidity and Mortality \\
\hline RTIS & Reproductive Tract Infections \\
\hline SRH & Sexual and Reproductive Health \\
\hline STIs & Sexually Transmitted Infections \\
\hline STD & Sexual transmitted Disease \\
\hline WHO & World Health Organization \\
\hline
\end{tabular}




\section{INTRODUCTION}

\section{Background}

In Ghana, in the absence of systematic national data, it is widely believed that abortions are a leading cause of maternal mortality. With a maternal mortality ratio of 540 per 100,000 live births (World Health Organization, 2006), efforts aimed at improving maternal survival need to pay particular attention to unwanted pregnancy and abortion. Hospital studies confirm that complications of abortion are a leading cause of gynecological admissions. A case- review of hospital admissions during the calendar year 2000 at the Korle-Bu Teaching Hospital (Ghana's largest teaching hospital) found that 41 percent of admissions were due to complications of abortion (Srofenyoh and Lassey, 2003). Poorly conducted induced abortions result in complications and sometimes death. In this hospital study, 14 percent of the maternal deaths recorded were due to abortion complications. Another review of hospital data from the KassenaNankana district validates that complications of abortion are an important reason for hospital admission and maternal death (Baiden et al., 2006). Analysis of hospital records of admissions between January 2000 to December 2003 found 24 maternal deaths to yield a hospital maternal mortality ratio of 759 per 100,000 livebirths. Seven of the 24 deaths were due to complications of abortion. The authors also suggest that these estimates are an under-estimate since clinical experience indicates "a high incidence of backstreet and unsafe practices."

Since 1985, Ghanaian law allows abortion to save a woman's life and to protect her mental and physical health. Also, abortion is allowed if the pregnancy occurred from rape or incest, or if continuation of the pregnancy results in physical abnormality or disease for the child. However, translation of the law into effective services has been limited. Abortion services tend to be available in large tertiary institutions and in urban areas, and less so in rural areas and in lessdeveloped regions of the country. In 2004, 1 in 10 regional hospitals, 41 percent of district and other hospitals, and 12 percent of lower level facilities reported providing post-abortion care (GHS and QHP, 2005). It is not clear to what extent providers understand the provisions of the abortion law and the specific conditions under which it may be offered. In addition to the limited service availability, many Ghanaians are unaware of their legal rights to abortion and where to access safe abortion services. 
Reliable data on induced abortions in Ghana is scarce. Since knowledge of the abortion law and its nuances is low in communities and there is sensitivity around abortions (both miscarriages and induced abortions), it is difficult to derive reliable community level estimates. Data gathered from facilities have limitations of incompleteness or inaccuracy; they also reflect the experiences of only those women who have sought services and not others. Lack of complete understanding of the abortion law makes providers unwilling to accurately classify the type of abortion (induced or spontaneous) and for women seeking care to admit to having induced an abortion.

Data from a large survey covering 18,301 women aged 15-49 from four of the ten regions in Ghana provide some estimates of the extent of abortions in the country (Ahiadeke, 2001). The study reported 19 induced abortions per 100 pregnancies or 27 abortions for every 100 live births, and the researcher also indicates that these estimates may yet be lower than the reality. Sixty percent of the women who had the abortions were younger than 30 and 36 percent were nulliparous. Very few had sought the service from a physician: 12 percent had received services from a physician and a large number of abortions were sought outside the medical system. Nearly half of the women had self-induced abortions or sought services from pharmacists. These data suggest that induced abortions are fairly common and women seek care from providers who may be untrained and conduct unsafe and unhygienic procedures.

While data on induced abortion is limited, other data on reproductive behaviors support the conclusion that induced abortions are widespread. According to the 2003 Demographic and Health Survey (DHS) there is considerable unwanted pregnancy-Ghanaian women have on average 4.4 children but the wanted fertility rate is 3.7. There is also a wide gap between contraceptive knowledge and use. While there is near universal knowledge of at least one modern method of family planning, use is low at 19 percent. ${ }^{1}$ Further, nearly a third of women (34\%) report an unmet need for contraception - (i.e.) where they wish to prevent a pregnancy in the short or long term but are not using contraception.

Taking into account different methodologies and study populations, the expert consensus is that at least a fifth of maternal deaths are due to induced abortions. Recognizing that induced

\footnotetext{
${ }^{1}$ Emergency Contraception was introduced in 1996; yet as of 2003, only 28 percent of women reported knowledge of the method.
} 
abortions are widespread in Ghana and that a high proportion of them are conducted in an unsafe manner, the Ghana Health Service is interested in redressing the situation and taking steps to reducing abortion related morbidity and mortality.

Ghana Health Service is providing direction and guidance for extending services beyond postabortion care to include quality abortion services (as permitted by Ghanaian law) and strengthened contraceptive services. This package of services is called Comprehensive Abortion Care (CAC) and aims to provide: safe, accessible and affordable abortion services, management of abortion complications, and provision of family planning counseling and services. Standards and Protocols for delivery of CAC services have been developed and are beginning to be disseminated to health facilities. The standards and protocols provide guidance to service providers on the service package including how to offer safe abortion services and to clients as to where to seek care.

In 2006, a consortium of six agencies-EngenderHealth, Ipas, Macro International, Marie Stopes International, Population Council, and Willows Foundation-came together to provide technical and financial support to the Government of Ghana in the roll-out of CAC services. The consortium collaborates with the Government of Ghana in expanding women's access to modern family planning and comprehensive abortion care. The consortium's program titled "Reducing Maternal Morbidity and Mortality" or R3M aims to reduce unwanted pregnancy and severe complications and deaths caused by unsafe abortions. Marie Stopes International (MSI) has established a center of excellence in Accra that provides a range of sexual and reproductive health services (SRH) at affordable prices to urban populations. This center has been operational since April 13, 2007. A second centre was established at Ashaiman in the Tema Metropolitan area on May 24, 2007 to provide similar services ${ }^{2}$. Further, efforts are underway to identify and franchise other private reproductive health centers in Ghana.

Population Council, Ipas, and EngenderHealth along with other cooperating agencies have begun the process of disseminating national standards and protocol guidelines for service delivery in comprehensive abortion care, especially in public sector facilities. Fifty-seven Ghana Health

\footnotetext{
${ }^{2} \mathrm{~A}$ third center is planned in the city of Kumasi.
} 
Service (GHS) service delivery sites have been chosen to provide comprehensive abortion care. Macro International is currently conducting a maternal mortality survey on the causes of maternal death, women's perceptions and experiences with antenatal, maternity, abortion and post abortion care. Ipas is also in the process of conducting a study into the quality of services received by clients seeking post-abortion and comprehensive abortion care in all public facilities that currently offer these services using client exit interviews.

The formative research reported in this document was undertaken to provide a profile of beneficiaries, their needs for information and services, their decision-making processes to seek care and service points, and the quality of care they received. The findings from the research are expected to guide health service institutions in the design and implementation of client oriented services and to support the R3M program in its effort to develop strategies and approaches for reducing maternal morbidity and mortality.

\section{Research Objectives}

The principal goal of the research is to generate evidence and information that could be fed into ongoing policy and program initiatives to expand access to legal and safe abortion services in public and private facilities. The specific objectives are:

1. To develop a profile of abortion seekers who visit public and private sector health facilities;

2. To better understand the decision-making processes that abortion seekers undergo; and

3. To document the quality of abortion services offered at private and public health facilities.

\section{ReSEARCH Methodology}

\section{Study Design}

A facility-based design was used and includes four health facilities where abortion services are

offered. Two of these are public sector facilities located in the Greater Accra region (Ridge and La General Hospitals) and two are private-sector facilities run by Marie Stopes International (MSI) - the Marie Stopes Centre, Ashaiman and the Marie Stopes Centre, Kokomlemle, also in the Greater Accra region. The inclusion of both public and private sector facilities is aimed at capturing a wide range of clients and client experiences. 


\section{Data Collection}

Four different types of data were collected: a review of patient case records maintained at the participating facilities, interviews with providers who provide abortion services at the facilities, observations of the interaction between providers and clients during the period of consultation and, client exit interviews. The research was conducted over a four-week period from November 5, 2007 to December 7, 2007.

Patient Case Records: The purpose of reviewing these records was to provide a description of abortion seekers (age, parity, place of residence, gestation), the type of service they received at the facility (type of evacuation-MVA, EVA, D\&C), complications, and caseloads in terms of volume and seasonality. In facilities where abortion services had been provided for longer than a year prior to the research, data were collected for the 12 month period preceding; for facilities that had been providing services for less than a year, data were collected from inception of services.

Provider interviews: Short structured interviews were conducted with providers to assess their training, current technical qualification, knowledge of standards and guidelines for comprehensive abortion service provision, types of abortion cases/complications seen, types of procedures performed at the facility, knowledge about the procedures as well as their knowledge of the law on abortion in Ghana. The interviews targeted all service providers in the four facilities. A total of 12 service providers were successfully interviewed.

Provider-client interaction: To obtain a measure of the quality of services provided to abortion clients, two provider-client interactions were observed daily in each facility during the study period. Specific service areas observed included: privacy and confidentiality of services, history taking, counseling, physical assessment of client, and the type of infection prevention procedures adopted. The consultations were observed by trained midwives and recorded on a standardized checklist. Only providers and women consenting to have their consultations observed were included in this part of the study. In all, 145 consultations were observed in the four facilities.

Client Exit Interviews: Clients who sought abortion services at the four facilities during the study period were interviewed after they had undergone the procedure and had recuperated. The interviewer collected information on background characteristics (i.e., education, economic status, 
urban/rural residence age, parity); the circumstances surrounding the pregnancy (non-use of contraceptives, contraceptive failure, timing, sexual coercion and/or rape); reasons for not wanting the pregnancy (economic, sexual coercion; too many children); care seeking process (previous referral points, knowledge of other sources, social support), and quality of care received (reception, information, medication, and inter-personal relations).

The clients were interviewed after they had recuperated. Recuperation is defined as the time when the client was ambulatory and after the nursing staff has checked her vital signs in preparation for her discharge. Interviews were conducted at least 30 minutes post procedure. Efforts were made to reduce the duration of the interview to 20 minutes or less so as to minimize the stress on the women post-procedure. Only clients who had undergone an abortion in the reference period and were willing to be interviewed were included. 146 abortion clients were interviewed in the four-week study period.

Twelve field staff were recruited and given four days of training on the objectives of the research, types of data to be collected, tools for data collection, importance of quality control, and ethical procedures to be followed. Following the training, the participants were split into four data collection teams, comprising of three members each (a trained midwife, a social scientist and a supervisor). The trained midwife was responsible for the observation of the provider client interactions and provider interviews while the social scientist conducted exit interviews with clients. The supervisor was charged with compiling data from the patient case records, checking completed questionnaires in the field for consistency and errors, and for monitoring the day-to-day activities of team members. Each data collection team was stationed at the assigned facility for four weeks. Prior to the data collection, permission was sought from the relevant management of the Ghana Health Service and heads of the four participating study facilities.

\section{Ethical Considerations}

Informed consent of all persons involved in the study was sought prior to the start of an interview or observation. In the case of data retrieved from patient case records, permission was taken from the supervisors of the participating facilities. The data were recorded on-site and returned to the supervisor at the end of each work day. Before providers were interviewed, their informed consent was sought. In the case of observations of client-provider interactions, permission was sought 
from both the provider and client before the consultation and the commencement of the data collection. A two-step consent process was followed to obtain consent from the client. First, a provider asked the client about her willingness to participate in the research. Clients who agreed were then approached by the interviewer and asked if they were willing to be interviewed. In the case of client exit interviews, only those clients who were willing to be interviewed after they had recuperated and recovered sufficiently from the procedure were included.

Recognizing the social stigma surrounding abortions and the sensitivity of the topic, the research team took steps to minimize discomfort to clients and providers by being unobtrusive to protect the privacy of the client, conducting interviews in privacy and of keeping in strict confidence any information gathered during the consultation and interview process. In order to ensure anonymity and confidentiality of responses, names and addresses of clients and providers were also not collected. Clinic level data collected through observations have also been kept in strict confidence to offer a layer of protection to providers. Each field staff was also required to sign an undertaking not to share any information gathered with anybody other than for the purpose of obtaining guidance on how to collect and record the data. All completed questionnaires and data recording sheets were stored in locked cabinets.

\section{FINDINGS}

\section{Type and Seasonality Pattern of Abortions}

In order to get a sense of the seasonality pattern of abortion, data on caseloads were extracted from patient case records at each facility. A total of 785 cases were obtained from the public facilities while 416 cases were extracted from the MSI data set for the seasonality analysis. Data

from the private facilities covers the period April to December 2007 as the first facility was established in April 2007.

Of the four facilities studied, only La General Hospital could provide relatively complete information on the type of services sought by clients who had sought services at the facility. Of the 394 abortion cases recorded at La General Hospital between November 2006 and October 2007, 372 (94\%) had information on type of services sought by the clients. The data suggest that there is an even distribution of clients for post-abortion services and elective abortions-50 
percent were recorded as having sought PAC services and the rest as having sought comprehensive abortion care.

Figure 1 below suggests that the cases of abortions recorded by the public health facilities (Ridge) were much higher in March, August and October 2007 than other months. The pattern exhibited by the private facilities (Kokomlemle and Ashaiman) shows a gradual monthly increase in the number of abortion cases until October when the curve began to dip. As with new establishments, the initial consistent increase in the number of cases reported at the private facilities is to be expected as people are still getting to know the existence of these facilities. The sudden unusual decline in the number of cases in November can, however, be attributed to the fact that one provider had to go on a two-week leave, which meant that arrangements had to be made for clients to receive care from a private consultant who visited the facility at scheduled periods. Clients who could not wait for the consultant may have sought services elsewhere.

\section{Figure 1: Distribution of recorded abortion cases by month of visit and type of sector}

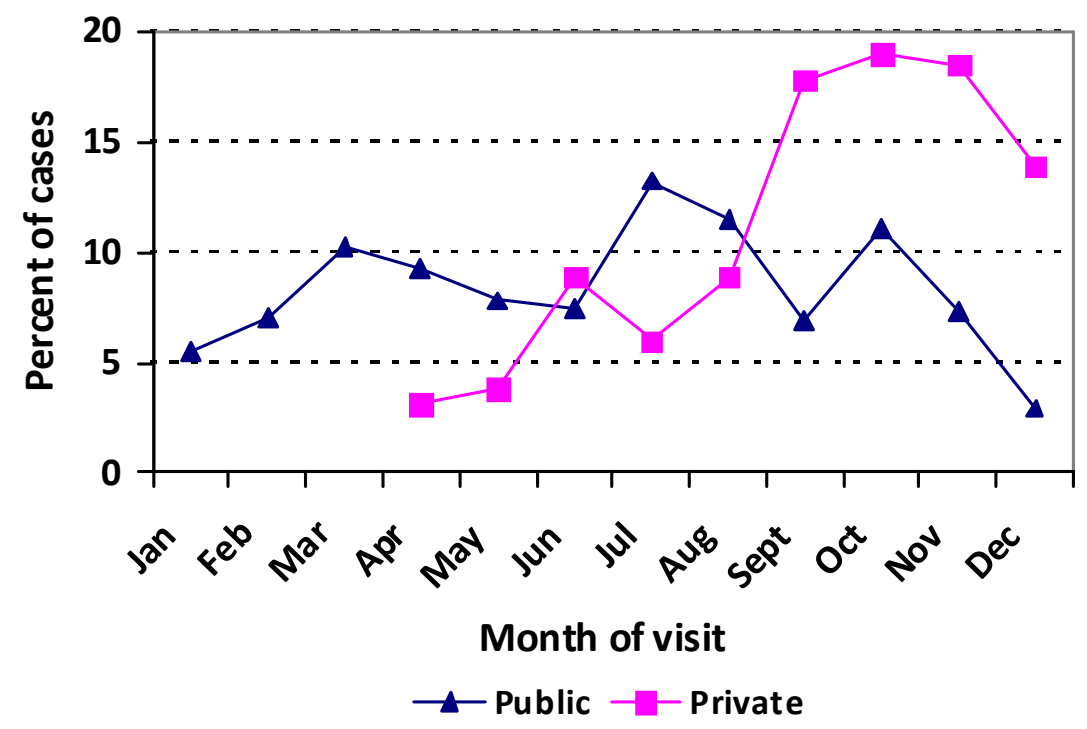

Source: Patient Case Records

Similarly, information on the type of complications presented by clients was complete only for Ridge Hospital. Of the 473 clients seen at this facility between 2006 and 2007, the following was recorded: women with heavy bleeding (56\%), threatened abortion (14\%), incomplete abortion 
(8\%), missed abortion (5\%), and ectopic pregnancy (2\%); the rest received comprehensive abortion care.

\section{Services Offered at Facilities}

Various type of abortion services are offered in the four study facilities. Most abortions are done by Manual Vacuum Aspiration (MVA) or medication abortion. Nine (75\%) of the interviewed providers reported that they offered MVA services and 8 (67\%) offered medication abortion. Six $(50 \%)$ reported that they offered both (MVA and medication abortion). Electric Vacuum Aspiration (EVA), Dilatation and Evacuation D\&E), Dilatation and Curettage (D\&C) were offered but only by a small proportion of the providers (Table 1). However, abortion with forceps was not reported. When asked about some of the abortion complications seen in the facility, 9 (75\%) providers mentioned excessive bleeding and sepsis, 2 (17\%) mentioned intra-abdominal or pelvic injury, and 1 mentioned poisoning.

Table 1: Types of abortion services offered by type of sector

\begin{tabular}{l|l|l}
\hline \multirow{2}{*}{ Services offered in facility } & \multicolumn{2}{|l}{ Number of providers reporting } \\
\cline { 2 - 3 } & $\begin{array}{l}\text { Private Sector } \\
\mathbf{( N = 3 )}\end{array}$ & $\begin{array}{l}\text { Public Sector } \\
\mathbf{( N = 9 )}\end{array}$ \\
\hline Medication abortion & 2 & 6 \\
Electric vacuum aspiration & 0 & 1 \\
Manual vacuum aspiration & 2 & 7 \\
Dilatation and evacuation & 1 & 3 \\
Dilatation and curettage & 0 & 2 \\
Forceps & 0 & 0 \\
\hline
\end{tabular}

Source: Provider Interviews

In terms of types of services offered at the facility, $11(92 \%)$ of the providers reported that they were offering CAC, 10 (83\%) were providing PAC services. However, it is not clear from these data whether providers clearly distinguish between the different service components of the CAC and PAC services.

\section{Provider Characteristics}

12 service providers were interviewed, 9 from the public sector facilities and 3 from the private sector. In terms of professional training, 7 were registered nurses with midwifery skills, 3 were 
medical officers and 2 were registered midwives. All providers reported providing PAC services, and 11 of the 12 reported providing CAC services as well. Since PAC is emergency management and treatment that has been traditionally offered in Ghana, it is not surprising that all providers reported providing PAC services. Among those providing CAC services, 4 providers had provided CAC for less than one year, 4 for more than 1 year, and 2 for more than 10 years.

\section{Pre-service Training and Knowledge of Service Guidelines and Abortion Law}

Most providers in both public and private sectors reported that they had received pre-service training on some aspect of abortion care during their pre-service training. Nine (75\%) reported that they had received training on the providing abortions, 10 (83\%) on Post Abortion Care (PAC), 8 (67\%) on counseling/behavior change communication on dangers of unsafe abortion, $10(83 \%)$ on counseling on pain management and $8(67 \%)$ on family planning.

Providers had sufficient understanding of the abortion law in Ghana. For example, most providers were able to express in their own words the conditions under which it could be offered lawfully and the procedures for informed consent. The 2007 Ghana Health Sector programme of work further stipulates that "the sector will implement high rapid delivery strategies that will sustain high coverage, remove barriers (physical and financial) to reproductive health care to ensure the right to attain the highest standard of sexual and reproductive health". ${ }^{3}$ To this end, the health sector has, as part of its annual programme of work outlined activities that aim at providing comprehensive abortion care within the context of the law. One would thus expect all providers to be conversant with this law as a requirement for providing abortion care services. When interviewed, however, two out of the $12(16.7 \%)$ providers indicated that they did not have any specific knowledge about the law. We infer that even though providers have a good understanding of the law, the data collection and interview format encourages reporting of knowledge gaps. When asked how well they knew the Ghana Standards and Protocols on Comprehensive Abortion Care, 4 (33\%) of the 12 staff who routinely provide abortion care knew these guidelines very well. As can be seen from Figure 2, knowledge on the specifics of CAC is limited. Only one provider had a detailed knowledge about post-procedure care and management of medication abortion while 3 (25\%) had detailed knowledge in pre-procedure care

\footnotetext{
${ }^{3}$ Ministry of Health, 2007. The Ghana Health Sector Annual Programme of Work, pages 13-14.
} 
and complication of medication abortion. Given that medication abortion is offered at all four facilities, it is clear that the level of knowledge about this procedure is low.

Figure 2: Provider's knowledge of CAC services by type of facility

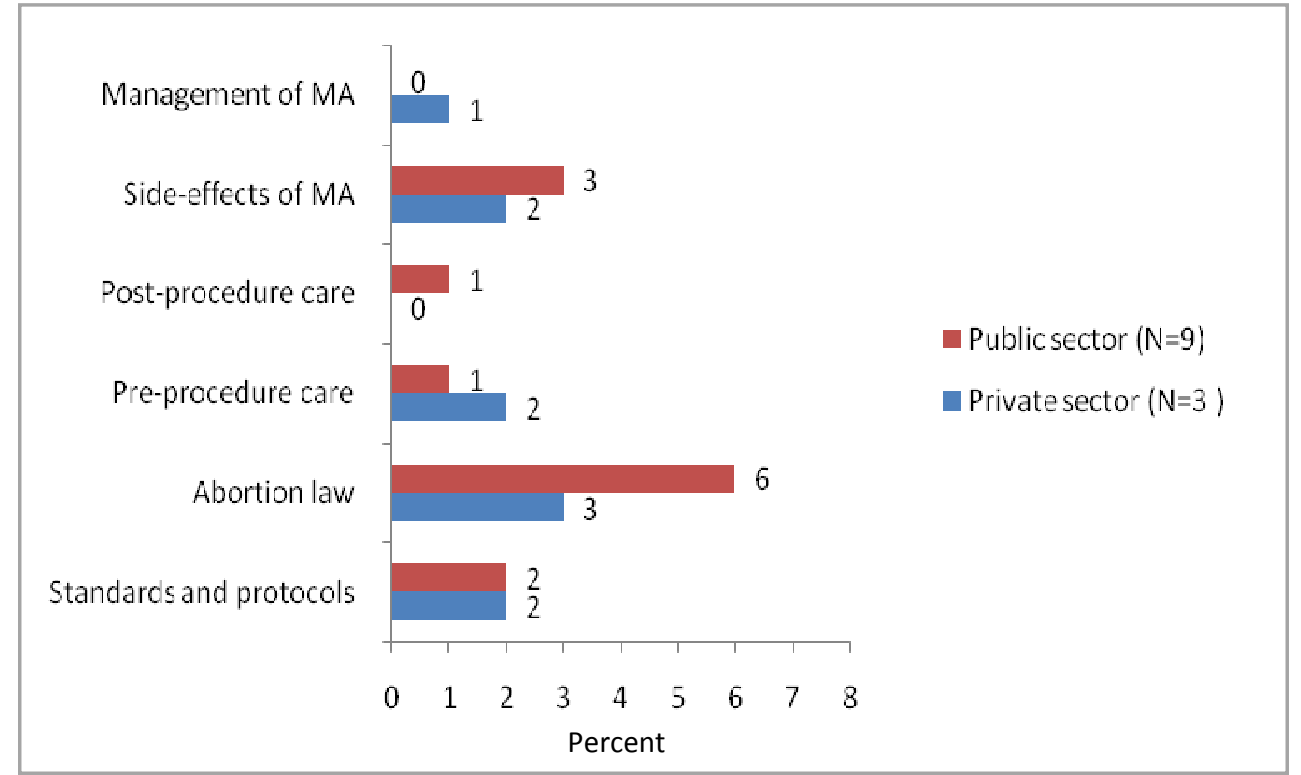

Source: Provider Interviews

\section{Professional Needs of Providers}

The three most important issues that providers felt needed to be addressed were: the lack of feedback on performance, better physical environment and equipment. In addition, 4 providers mentioned needing more training and better staff motivation. It is important to mention that all of those (8) who cited better physical environment were from the public sector. In terms of training needs, Table 2 shows that 11 of the 12 providers indicated a need for additional training in order to provide good quality CAC and PAC services.

Table 2: Provider training needs by type of facility

\begin{tabular}{l|l|l}
\hline Training needs of providers & Private sector & Public sector \\
& $\mathrm{N}=3$ & $\mathrm{~N}=9$ \\
\hline Refresher training & 1 & 4 \\
Regular orientation & 1 & 1 \\
Upgrade or top up courses & 0 & 3 \\
Others & 1 & 1 \\
\hline
\end{tabular}

Source: Provider Interviews 


\section{Quality of Care Given During Provider-client Interaction}

A total of 145 consultations were observed in the four facilities ( 77 from private and 68 from public). According to the $\mathrm{WHO}^{4}$, the first step in providing abortion care is to establish rapport with the client followed by comprehensive history-taking, including personal and family history of relevant diseases, current use of medication, history of sexually transmitted infections and obstetric and gynecological history. We present the results along this same schema.

Providers were observed on different elements of rapport creation and interpersonal communication such as being patient with the client, using language that clients can understand privacy and confidentiality. In general, rapport with clients was good across all the facilities. Observations of the consultations indicated that providers from the private sector facilities interacted better with clients than those from the public sector on many items of care giving (Figure 3). In particular, issues of privacy and confidentiality were not addressed in the public sector to the same extent as in the private sector. These may reflect differences in training of public and private sector providers as well as availability of physical space.

Figure 3: Interpersonal communication by type of facility

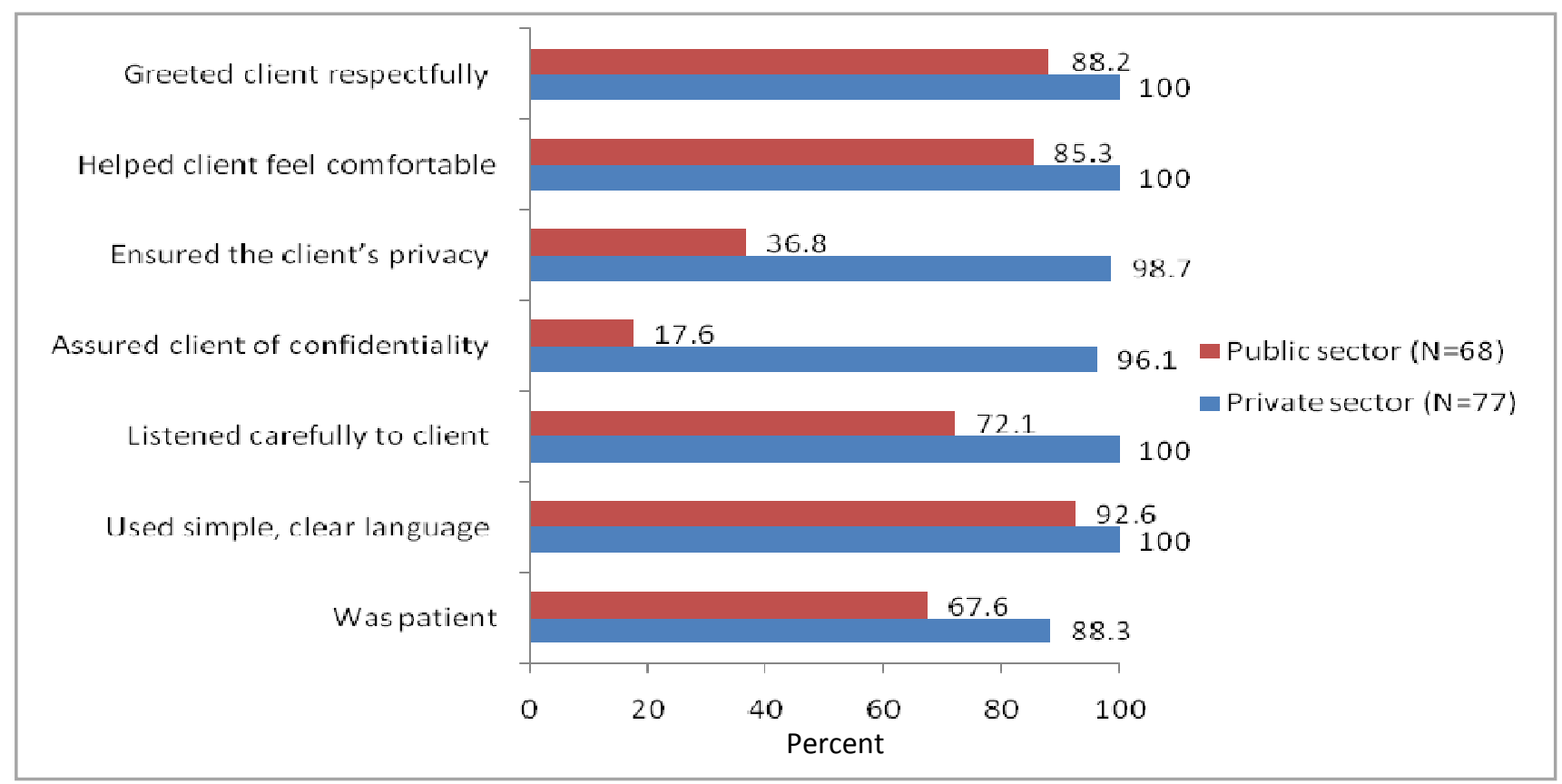

Source: Provider-Client Interaction

\footnotetext{
${ }^{4}$ Safe Abortion: Technical and Policy Guidance for Health Systems, WHO, Geneva, 2003.
} 


\section{Comprehensive History-taking}

History-taking is an important part of the consultation process where the provider engages in a conversation with the client and elicits information that are relevant in the management and treatment options to be considered. History-taking includes information on personal and family history of relevant diseases, current use of medication, history of sexually transmitted infections, obstetric and gynecological history, and some relevant social factors. Table 3 shows that the consultations in public sector facilities fared poorly against those conducted in the private facilities. For example, an important step in the management process is determining if the client is pregnant, and the gestational age based on the client's reports of her last menstrual cycle; in particular, the WHO highly recommends that the client should be asked about the first day of her last menstruation period ${ }^{5}$. In over a tenth of the consultations in the public sector $(13.2 \%)$, clients were not asked the date of their last menstruation compared to all in the private sector. Furthermore, in both the public and private facilities, consultations did not cover the history of previous abortions (14\% in private and $68 \%$ in public); information on contraceptive history was asked in less than a fifth of the consultations in the public sector (19\%) compared to over 90 percent in the private facilities. Knowing if a client has had abortions in the past and her contraceptive history are important as it highlights the extent to which the client needs contraceptive counseling and services so that she can avoid an unwanted pregnancy in the future. Consultations in the public sector rarely collected detailed medical histories of the clients unlike the private sector.

\footnotetext{
${ }^{5}$ Conclusions of an International Consensus Conference on Medication Abortion in Early First trimester, 2006,Bellagio, Italy
} 
Table 3: History-taking during consultation by type of facility

\begin{tabular}{|c|c|c|}
\hline Percent of observations where provider asked client : & $\begin{array}{l}\text { Private Sector } \\
(\mathrm{N}=77)\end{array}$ & $\begin{array}{l}\text { Public Sector } \\
(\mathrm{N}=68)\end{array}$ \\
\hline $\begin{array}{l}\text { Reasons for visit and details of the complaints } \\
\text { Reasons for termination of the pregnancy } \\
\text { Details of the complaints }\end{array}$ & $\begin{array}{l}100.0 \% \\
93.5 \%\end{array}$ & $\begin{array}{l}85.3 \% \\
36.8 \% \\
\end{array}$ \\
\hline $\begin{array}{l}\text { Obstetric History: } \\
\text { Parity } \\
\text { Outcome of previous pregnancies } \\
\text { Abortion }\end{array}$ & $\begin{array}{l}98.7 \\
64.9 \\
85.7\end{array}$ & $\begin{array}{l}82.4 \\
26.5 \\
32.4\end{array}$ \\
\hline $\begin{array}{l}\text { Menstrual History } \\
\text { Date of last menstrual period } \\
\text { Menstrual cycle (pattern?) }\end{array}$ & $\begin{array}{l}100.0 \\
98.7\end{array}$ & $\begin{array}{l}86.8 \\
33.8\end{array}$ \\
\hline $\begin{array}{l}\text { Medical History } \\
\text { Hypertension } \\
\text { Seizure disorder } \\
\text { Blood clotting disorders } \\
\text { Heart disease } \\
\text { Sickle cell disease } \\
\text { Asthma } \\
\text { Past hospitalization } \\
\text { Past surgical operations } \\
\text { Recent medications taken, including misoprostol and herbal } \\
\text { Allergy to medications }\end{array}$ & $\begin{array}{l}71.4 \\
98.7 \\
98.7 \\
72.7 \\
70.1 \\
98.7 \\
98.7 \\
98.7 \\
92.2 \\
98.7\end{array}$ & $\begin{array}{l}1.5 \\
0.0 \\
0.0 \\
1.5 \\
2.9 \\
1.5 \\
0.0 \\
0.0 \\
20.6 \\
1.5\end{array}$ \\
\hline $\begin{array}{l}\text { Contraceptive History } \\
\text { Current contraceptive use } \\
\text { Type of contraceptive used } \\
\text { Reason for non-use for those not using }\end{array}$ & $\begin{array}{l}90.9 \\
55.8 \\
67.5\end{array}$ & $\begin{array}{l}19.1 \\
10.3 \\
13.2\end{array}$ \\
\hline $\begin{array}{l}\text { Sexual History } \\
\text { Number of sexual partners } \\
\text { History of RTIs, STIs and HIV/AIDS }\end{array}$ & $\begin{array}{l}71.4 \\
6.5\end{array}$ & $\begin{array}{l}0.0 \\
0.0\end{array}$ \\
\hline $\begin{array}{l}\text { Social History } \\
\text { Marital status } \\
\text { Family environment and support system } \\
\text { Experience of violence }\end{array}$ & $\begin{array}{l}84.4 \\
24.7 \\
11.7\end{array}$ & $\begin{array}{l}76.5 \\
52.9 \\
0.0\end{array}$ \\
\hline
\end{tabular}

Source: Provider-Client Interaction

\section{Quality of Examination Conducted During Consultation}

Table 4 presents data on the extent to which providers were observed to conduct different aspects of the physical examination. The most critical actions highly recommended by both WHO and the Ghana standards on Comprehensive Abortion Care are: a speculum exam, assessment of the size of the uterus, and confirming pregnancy with a pregnancy test. These three actions were not 
only more likely to occur in consultations in the private sector than in the public sector facilities but also at much higher levels: a speculum exam (94\% in private compared to $40 \%$ in public), assessment of uterus ( $87 \%$ in private compared to $44 \%$ in public), and doing or referring a pregnancy tests (98\% in private compared to $50 \%$ in public). From these data it is not clear whether the public sector facilities do not routinely conduct these activities because of lack of knowledge of protocols, lack of equipment, or providers are hard-pressed for time due to heavy case-loads. As can be seen from Table 4, consultations in the private sector tend to be of higher quality in most other aspects of the care giving process as well; for example, different aspects of the speculum exam were done in at least four-fifths of the consultations in the private sector compared to less than two-fifths of the time.

Table 4: Percent of observations in which various examinations were performed by type of examination and sector

\begin{tabular}{l|l|l}
\hline Examinations/procedures performed by provider & \multicolumn{2}{l}{ \% of observations } \\
\cline { 2 - 3 } during consultation & $\begin{array}{l}\text { Private Sector } \\
\text { N=77 }\end{array}$ & $\begin{array}{l}\text { Public Sector } \\
\text { N=68 }\end{array}$ \\
\hline Physical Exam & 100.0 & 54.4 \\
Took blood pressure & 94.8 & 29.4 \\
Took pulse & 32.5 & 42.6 \\
Checked for signs of anemia & & \\
\hline Speculum Exam & 93.5 & 39.7 \\
\hline Conducted speculum exam & 87.0 & 14.7 \\
Explained speculum exam procedure & 96.1 & 39.7 \\
Ensured visual privacy & 97.4 & 29.4 \\
Ensured good lighting & 96.1 & 4.4 \\
Asked client to empty bladder before hand & 88.3 & 8.8 \\
Used linens or drapes to protect client's privacy & 97.4 & 33.8 \\
Reduced client's anxiety by verbal reassurances & & \\
\hline Bimanual Exam & 87.0 & 44.1 \\
\hline Assessed size of uterus & 93.5 & 7.4 \\
Checked for signs of pregnancy & & 50 \\
\hline Lab Exams & 97.4 & \\
\hline Conducted or requested for a pregnancy test &
\end{tabular}




\section{Information and Counseling Given During Consultation}

The provision of information is an essential part of good-quality abortion services. Furthermore, counseling is very important in helping the woman consider her options and to ensure that she can make decisions free from pressure. Thus, quality of care was also measured in terms of counseling and information provided on the available methods of abortion, details of the procedure, and other pertinent information such as service fees.

Figure 4: Information and Counseling Given During Consultation

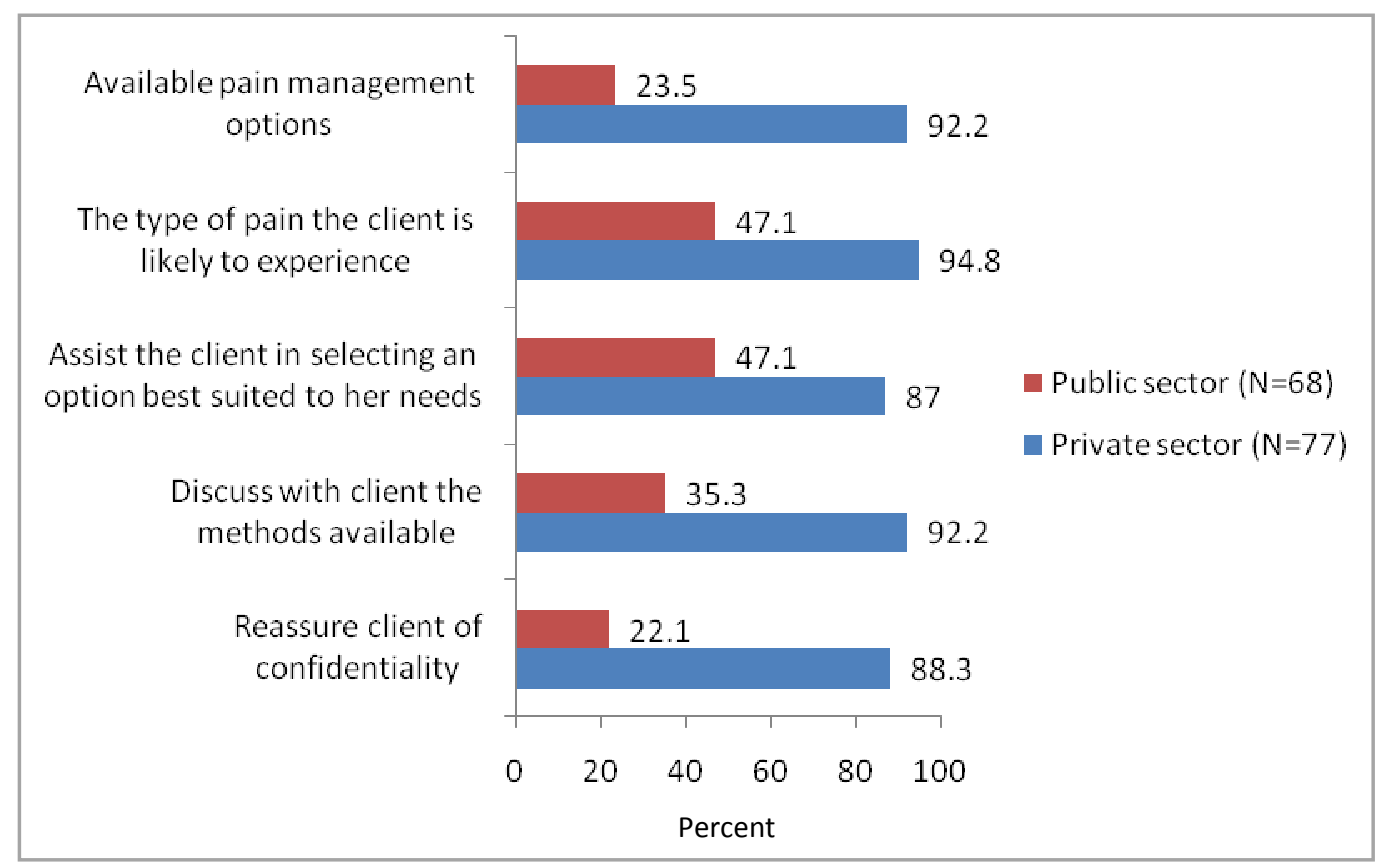

Source: Provider-Client Interaction

Information on return to fertility and contraceptive options is a crucial component of postabortion counseling as it provides a woman with the necessary tools to ways to prevent future unwanted pregnancies. Information provision on contraceptive options is the weakest part of the service package in the public sector, and is the strongest part in the private sector. Figure 4 indicates that on almost every aspect of contraceptive information and service provision, the private sector facilities far out-perform the public sector facilities. There are areas that need to be strengthened in both types of facilities. For example, over a fifth of the consultations in the private sector and less than one in ten consultations were clients informed that fertility could return within 10 days post-abortion. In addition, in at least a quarter of the consultations, clients are not asked about their fertility intentions ( $>=40 \%$ in public and $>=25 \%$ in private). Interesting 
enough even when contraceptive options and services are provided, information is not provided on methods that both protect against pregnancy and sexually transmitted infections.

Figure 5: Counseling on family planning by type of sector

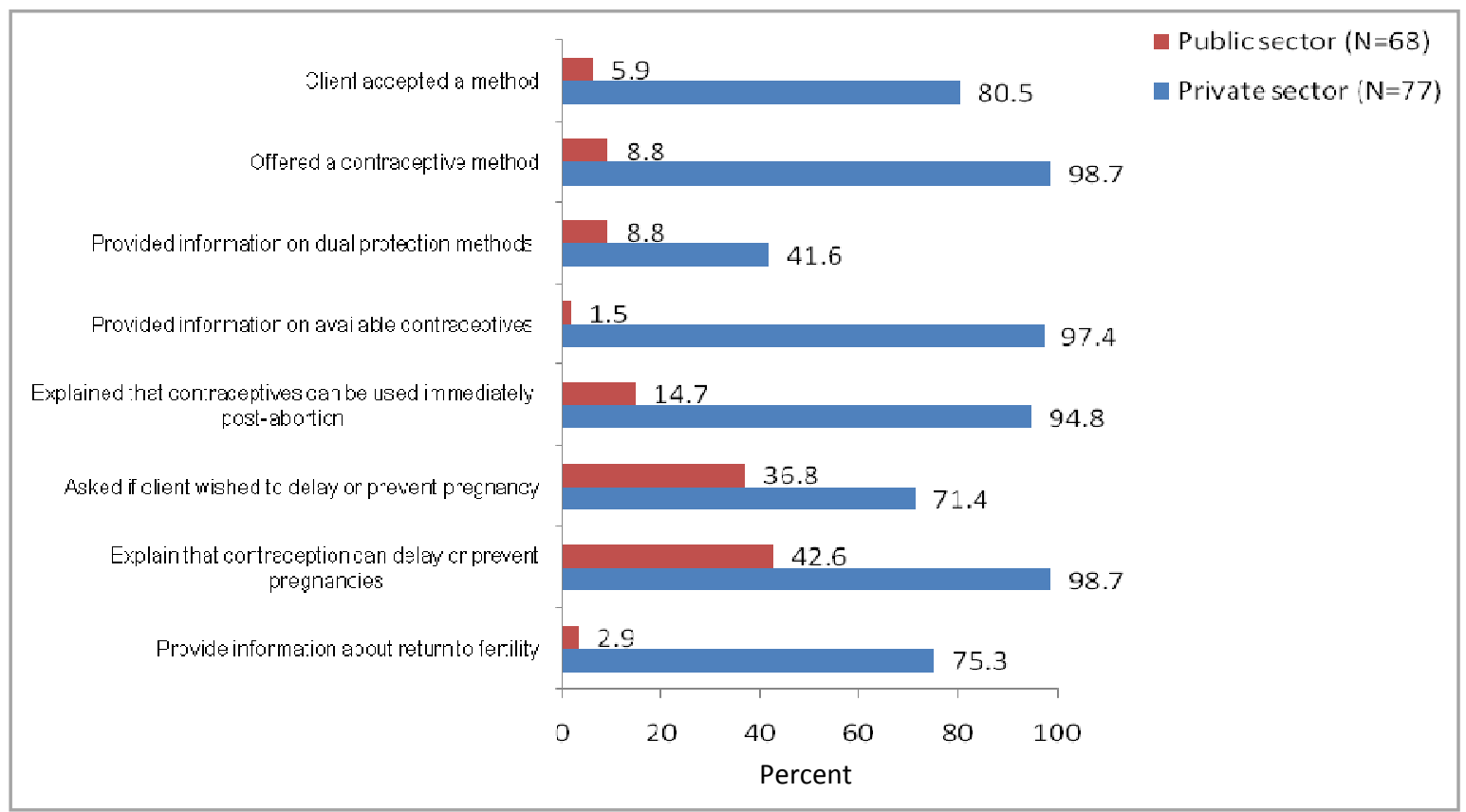

Source: Provider-Client Interaction

\section{Client's Background Characteristics}

Of the 146 clients interviewed, slightly more than half (52\%) had patronized the private facilities and as many as 38 percent had sought services at the Ashaiman MSI centre alone, while the Kokomlemle MSI centre recorded the lowest number of client visits (Table 5) .

Table 5: Percentage distribution of clients by type of sector and facility

\begin{tabular}{c|l}
\hline Type of Sector & $\%$ of clients \\
\hline Public & 47.9 \\
Private & 52.1 \\
\hline Type of Facility & $\%$ \\
La General Hospital & 21.2 \\
Ridge Hospital & 26.7 \\
MSI Kokomlemle & 14.4 \\
MSI Ashaiman & 37.7 \\
\hline Total (\%) & 100.0 \\
N & 146 \\
\hline
\end{tabular}

Source: Client Exit Interviews 
Table 6 shows the distribution of the clients by their background characteristics. The age of the clients ranged from 14 to 41 years, with a mean of 25 years. Nearly three-fifths (59\%) of the clients who had sought abortion or post-abortion care services within the study period were young adults: 41 percent in the age group of 20-24 and 18 percent were aged between 14 and 19 years. Those aged 35 years and over comprised 9 percent of the respondents.

86 percent of the clients had received some level of education; two-fifths (37\%) had had middle or junior secondary school education while about a third had received senior secondary or higher level education. A large proportion of the clients were engaged in sales (36\%) and service (23\%) occupations. Those in professional, technical, administrative and clerical occupations accounted for 10 percent while the unemployed (16\%) and students (12\%) made up 28 percent of the study sample. Clients accessing the two different sectors of health care were similar on most background characteristics except for their occupation. The data suggests that those who work in profession or technical jobs workers are more likely to seek care from private facilities-11 percent of those seeking care from private facilities were professional/technical workers and in comparison, no professional/technical worker sought abortion services at the public facilities. From these data it is not possible to conjecture if these patterns are indeed reflective of the true patterns of use since the time period of the study was limited to a month. With respect to ethnic affiliation, Akans were in the majority (37\%), followed by Ewes (25\%) and Gas (23\%).

Attempts were also made to find out where the clients were coming from to seek these services. The data suggest that about 71 percent of the clients lived in the same area or town where the facilities are located and just about a quarter (24\%) travelled from neighboring cities or towns. As earlier indicated, three of the study facilities were located in the city of Accra and one in the city of Tema. Clients travelling from rural areas to these health centres accounted for only 3 percent of the respondents. The research design adopted in this assessment does not allow us to remark on the accessibility of services at the community level which will include both users and non-users of services as well. 
Table 6: Percentage distribution of clients seeking abortion care by background characteristics and type of sector

\begin{tabular}{|c|c|c|c|}
\hline Characteristics & Public (\%) & Private (\%) & Total $(\%)$ \\
\hline \multicolumn{4}{|l|}{ Age group } \\
\hline$<20$ & 17.1 & 18.4 & 17.8 \\
\hline $20-24$ & 44.3 & 38.2 & 41.1 \\
\hline $25-29$ & 17.1 & 17.1 & 17.1 \\
\hline $30-34$ & 11.4 & 13.2 & 12.3 \\
\hline $35+$ & 8.6 & 9.2 & 8.9 \\
\hline Not Stated & 1.4 & 3.9 & 2.7 \\
\hline \multicolumn{4}{|l|}{ Level of education } \\
\hline None & 14.3 & 14.5 & 14.4 \\
\hline Primary & 18.6 & 18.4 & 18.5 \\
\hline Middle/JSS & 34.3 & 39.5 & 37.0 \\
\hline Secondary & 21.4 & 15.8 & 18.5 \\
\hline Higher & 11.4 & 11.8 & 11.6 \\
\hline \multicolumn{4}{|l|}{ Occupation** } \\
\hline Not working & 15.7 & 15.8 & 15.8 \\
\hline Professional/Technical & 0.0 & 10.5 & 5.5 \\
\hline Administrative & 4.3 & 1.3 & 2.7 \\
\hline Clerical & 0.0 & 3.9 & 2.1 \\
\hline Sales & 32.9 & 39.5 & 36.3 \\
\hline Services & 31.4 & 15.8 & 23.3 \\
\hline Manual & 1.4 & 2.6 & 2.1 \\
\hline Student & 14.3 & 10.5 & 12.3 \\
\hline \multicolumn{4}{|l|}{ Ethnicity } \\
\hline $\mathrm{Ga}$ & 22.9 & 23.7 & 23.3 \\
\hline Akan & 42.9 & 31.6 & 37.0 \\
\hline Ewe & 17.1 & 32.9 & 25.3 \\
\hline Dagomba & 5.7 & 3.9 & 4.8 \\
\hline Other & 11.4 & 7.9 & 9.6 \\
\hline Place of residence & & & $\%$ \\
\hline In this town & 77.1 & 64.5 & 70.5 \\
\hline In neighboring city/town/ village & 20 & 31.5 & 26.1 \\
\hline Another different city/town/ village & 2.9 & 3.9 & 3.2 \\
\hline \multicolumn{4}{|l|}{ Persons with whom client resides } \\
\hline One or both parents & 21.4 & 22.4 & 22 \\
\hline Husband/ Boyfriend & 31.5 & 21 & 26.1 \\
\hline Siblinde & 18.6 & 21 & 19.9 \\
\hline Brother & 2.9 & 1.3 & 2.1 \\
\hline Grandparents/ Other relatives & 15.7 & 19.7 & 17.8 \\
\hline Friends/Hostel & 1.4 & 2.6 & 2.1 \\
\hline Lives alone & 8.6 & 13.2 & 11.0 \\
\hline Other & 2.9 & 0.0 & 1.4 \\
\hline Total \% & 100.0 & 100.0 & 100.0 \\
\hline $\mathrm{N}$ & 70 & 76 & 146 \\
\hline
\end{tabular}

Source: Client Exit Interviews 
The nature of the living arrangements provides insight into the type of social support that is available to the woman as well as the possibility for privacy and confidentiality. Almost a quarter (24\%) of the clients lived with their husbands or boyfriends; almost a quarter with one or both parents $(22 \%)$, and over a third lived with other relatives such as siblings, grandparents and aunts (38\%), and nearly 15 percent lived independently.

\section{Maternity History and Personal Abortion Experiences of Clients}

For 32 percent of the clients, this was the first pregnancy they were carrying (Table 8). Among the 99 clients who had experienced an earlier pregnancy, 16 percent had no living children. The rest reported having between 1 and 6 living children with a mean of 1.6 children. 25 percent of the interviewed clients reported having experienced repeat induced abortions. The number of previously induced abortions ranged from 1 to 5 , with a mean of 1.5 . Of the 37 clients $(35.3 \%)$ who had previously experienced an induced abortion, 25 (68\%) had had just one abortion prior to the current one.

\section{Table 7: Distribution of clients by maternity history and induced abortion experience}

\begin{tabular}{l|l}
\hline Characteristic & Percent of clients \\
\hline Number of pregnancies including current one & $\mathrm{N}=146$ \\
$1-3$ & 74.7 \\
$4-6$ & 21.2 \\
$7+$ & 4.1 \\
\hline Number of living children among women who had been previously pregnant & $\mathrm{N}=99$ \\
0 & 16.4 \\
$1-3$ & 41.8 \\
$4-6$ & 9.6 \\
\hline Number of previous induced abortions & $\mathrm{N}=146$ \\
0 & 74.7 \\
$1-3$ & 24 \\
$4-5$ & 1.4 \\
\hline
\end{tabular}

Source: Client Exit Interviews

\section{Circumstances Surrounding Current Pregnancy}

When asked about the circumstances surrounding their abortion, over 90 percent of the respondents reported that both partners had a voluntary sexual relationship; however, a few women $(7 \%)$ reported that the pregnancy was the result of forced or unwanted sex or that they had been persuaded by their partners to have sex at a time when they were not prepared to do so. When asked about contraceptive use, 91 percent indicated that they had not used any method to 
prevent or avoid pregnancy. Among those who had used contraception, the methods used were the male condom $(3 \%)$, the pill $(3 \%)$, the injectable $(1 \%)$, and the female condom $(1 \%)$.

In order to understand women's decision-making processes, respondents were asked their reasons for deciding not to continue with the pregnancy. As can be seen from Figure 5 below, a majority of the respondents indicated that the pregnancy was either mistimed (40\%) or unwanted either by themselves, their partners or other family members $(20 \%)$. Others were concerned that they could not take care of the baby due to financial constraints (21\%), and a significant proportion felt that a pregnancy and a child would interrupt their work (16\%) or education (16\%). Other reasons given included relationship problems, being psychologically prepared or not being old enough to be a mother, and not being married.

Figure 6: Reasons given by clients for aborting current pregnancy

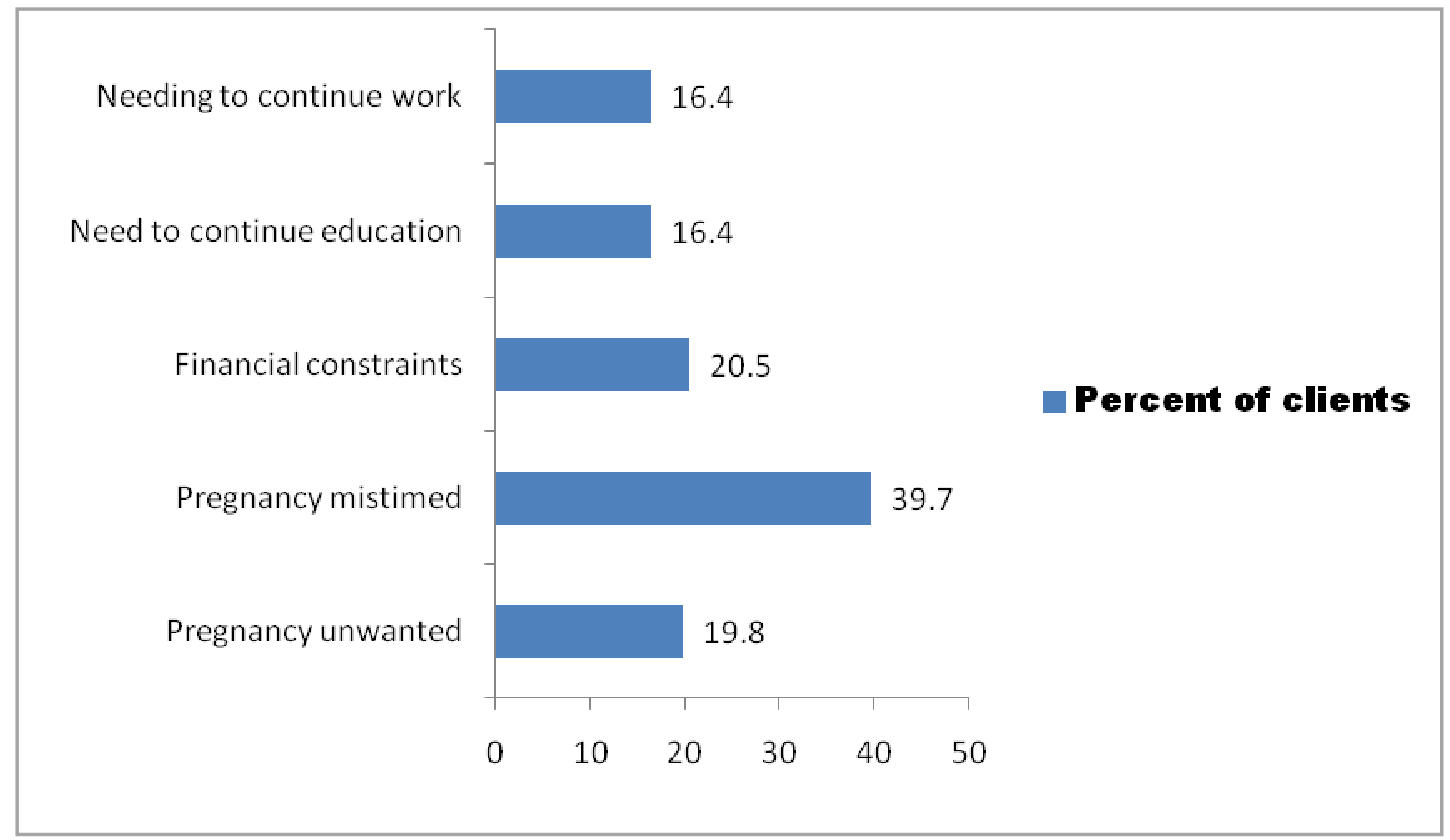

\section{Source: Client Exit Interviews}

Clients discovered that they were pregnant when the fetus was on average less than 5 weeks old (4.6 weeks). The pregnancy was mostly detected through a delayed or missed menstrual period (73\%), a pregnancy test (17\%), unusual vomiting or nausea (4\%) or experiences from a previous pregnancy (3\%). Others (4\%) went to see a doctor, had engorged breasts, skin rash, felt the pregnancy through their stomachs or were informed by their husbands that they were pregnant. 
Commonly expressed reactions on discovering the pregnancy include: unhappiness (57\%), worry (25\%), being scared (12\%), happiness (8\%), guilt (6\%), shock, surprise, or discomfort (3\%). The rest had no particular feelings (14\%). Respondents could provide more than one response to this set of questions.

\section{Social Support and Decision-making}

The assessment sought to examine whether the respondents had confided in their partners and others when they discovered that they were pregnant, and whether they received the desired support. About 93 percent (136) of the clients indicated that they had informed their partners when they realized that they were pregnant. Of the women who had discussed with their partners, 72 percent indicated that their partners had concurred with them to terminate the pregnancy; however, nearly a fifth $(21 \%)$ of the women reported that their partners had been opposed to the idea. The rest $(7 \%)$ indicated that their partners had neither agreed or opposed the abortion. When respondents were further asked about the extent to which their partners had been involved in the decision-making process, nearly four-fifths (79\%) reported receiving emotional support and money from their partners to have the abortion. To a much smaller extent, other women reported not receiving any type of support (7\%), that partners had been aware or were not involved in the decision to terminate the pregnancy $(6 \%)$, or that partners had claimed no responsibility for the pregnancy (4\%).

Women reported talking about the pregnancy and seeking support from a number of people. Approximately sixty percent $(60 \%)$ of the women indicated that they had informed other people besides their partners about the pregnancy. Typically, women tended to confide in their friends $(47 \%)$, sisters $(35 \%)$, mothers $(21 \%)$ and other relatives $(5 \%)$. Within the health system, study participants had discussed their pregnancy with health workers and had sought their opinions (5\%). Women were more likely to speak with their partners first about the pregnancy (83\%) while friends were the next likely group of people (10\%) to be consulted. Others reported talking first to family members such as their mothers (4\%), sisters $(2 \%)$, and other family members.

In terms of the final decision to terminate the pregnancy, nearly half the women (47\%) indicated that they had taken the decision by themselves, 34 percent reported that it was a joint decision between them and their partners, while 13 percent said the decision was solely taken by their partners. 
Figure 7: Person (s) who took the final decision to terminate the pregnancy

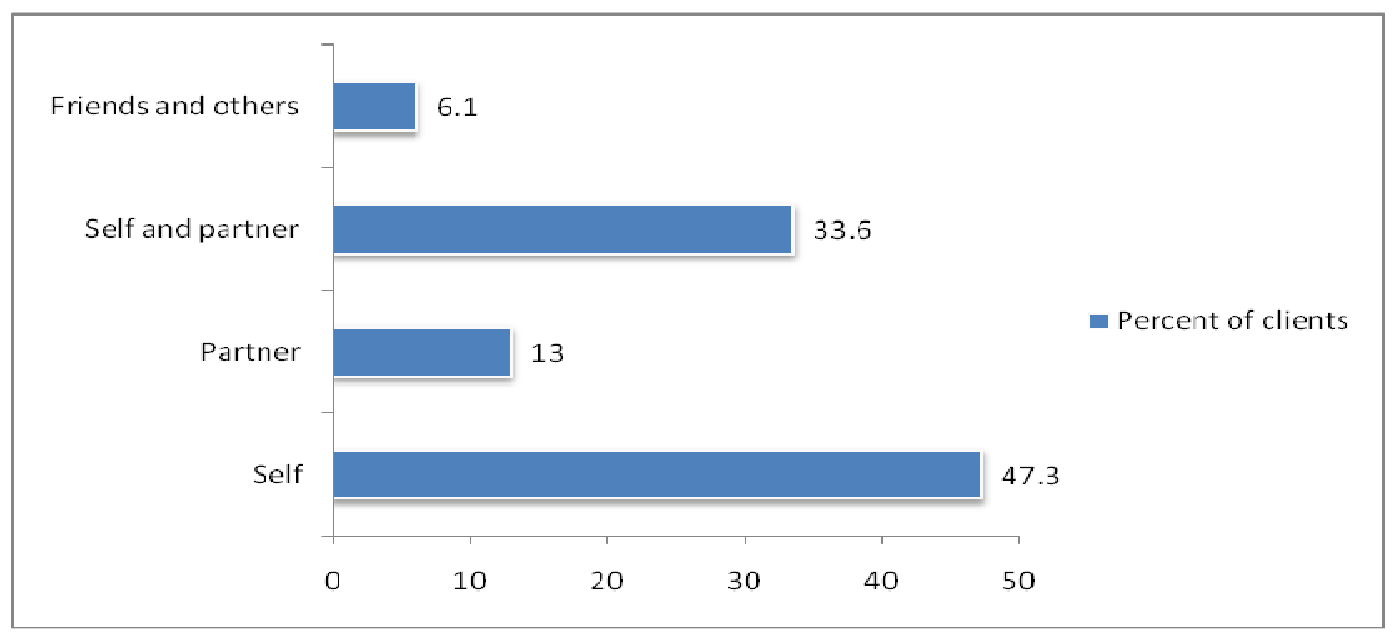

Source: Client Exit Interviews

Fifty-five women (38\%) had attempted to terminate the pregnancy on their own. Commonly reported ways of attempted terminations include: oral medications (75\%), home-made concoctions (18\%), inserting herbs into their reproductive tract (18\%), inserting medicines (15\%), drinking the beverage Guinness combined with either paracetamol or sugar (4\%). About one in ten women (8\%) reported that a family member or friend had tried to perform the abortion for them by giving oral medications. Women also reported seeking help from pharmacists and chemical sellers. Anecdotal evidence indicates that Cytotec is known among women and pharmacists to induce an abortion. Before arriving at the health facility for care, 17 percent of the women had visited pharmacy or chemical shops when the initial attempt to terminate the pregnancy had failed.

In terms of other social support received, nearly two-thirds of the women (62\%) reported a companion had accompanied them to the health facility. Companions are likely to be partners $(19 \%)$, sisters (15\%) or friends (13\%). However, nearly a third of the women had arrived at the health facility alone. 
Table 8: Distribution of clients by type of persons accompanying them to the facility

\begin{tabular}{l|l}
\hline Person accompanying client to the facility & $\begin{array}{l}\text { Percent of clients } \\
(\mathrm{N}=146)\end{array}$ \\
\hline No one & 31.5 \\
Partner (boyfriend/husband) & 19.2 \\
Sister & 15.1 \\
Mother & 9.6 \\
Other relative & 7.4 \\
Friend & 13.0 \\
Health worker & 4.8 \\
Mother-in-law/Boyfriend's mother/Boyfriend's sister & 3.5 \\
Neighbor & 0.7 \\
Stranger & 1.4 \\
Referred from another facility & 0.7 \\
\hline Sor Chie Exith
\end{tabular}

Source: Client Exit Interviews

\section{Client's Choice of Source of Care}

Most women reporting consulting with another person on deciding which health facility to seek services from although a significant proportion of women (32\%) indicated that they had chosen the facility themselves. Almost a quarter (24\%) mentioned that it was a joint decision between themselves and their partners. Overall, 55 percent of the women had a say in the decision to seek services at the current facility.

Figure 8: Person (s) who took the final decision to access abortion services at facility on the day of interview

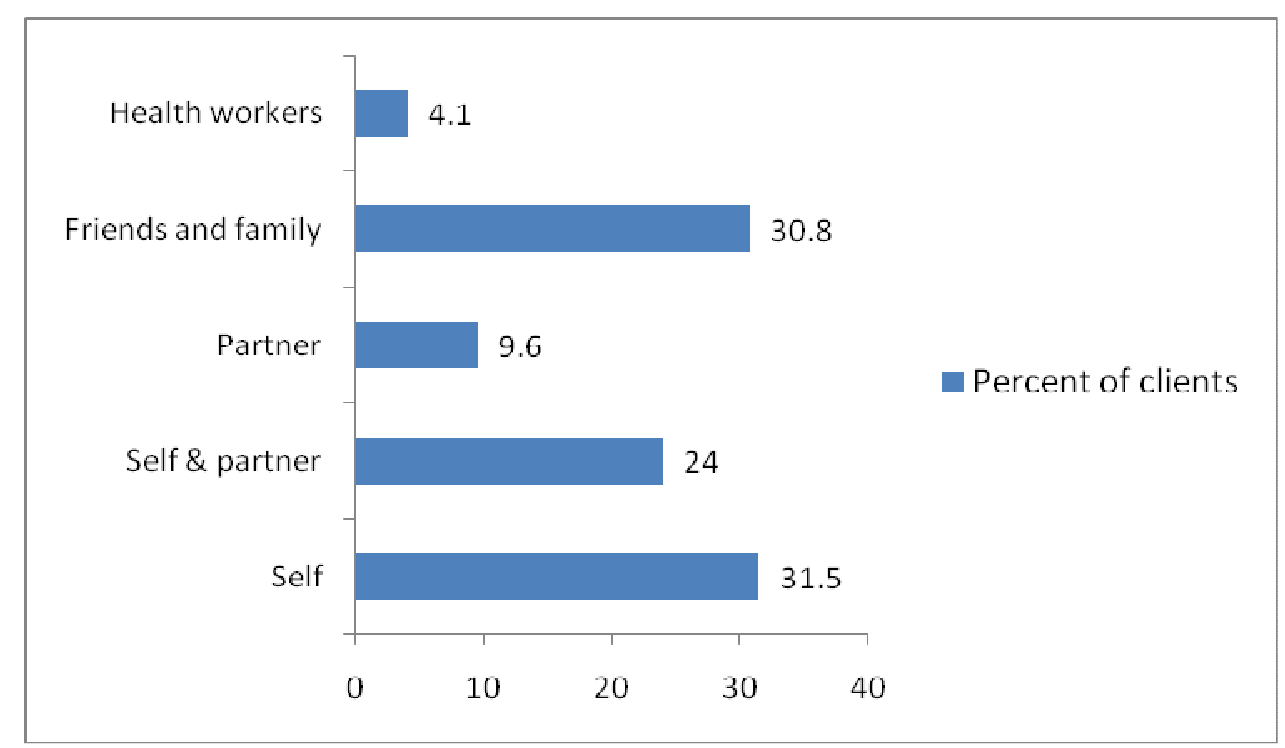

Source: Client Exit Interviews 
Table 10 indicates that over two-thirds (64\%), of the women reported that the facility had been recommended by others; others reported hearing of the facility's good reputation (19\%), or had been referred $(18 \%)$.

Table 9: Distribution of clients by reason for choosing facility for pregnancy termination

\begin{tabular}{l|l|l|l}
\hline \multirow{2}{*}{ Reason for choosing facility } & \multicolumn{3}{l}{$\begin{array}{l}\text { Percent of clients } \\
(\mathrm{N}=146)\end{array}$} \\
\cline { 2 - 4 } & Public & Private & Total \\
\hline Recommended by others & 37.1 & 89.5 & 64.4 \\
Good reputation (boyfriend/husband) & 27.1 & 10.5 & 18.5 \\
Client referred from another facility & 37.1 & 0.0 & 17.8 \\
Good quality & 5.7 & 3.9 & 4.8 \\
Access & 17.2 & 1.3 & 2.8 \\
Other attempts did not work & 1.4 & 0.0 & 0.7 \\
No special reason & 2.9 & 3.9 & 3.4 \\
Other & 2.8 & 0.0 & 1.4 \\
\hline Sourc:
\end{tabular}

Source: Client Exit Interviews

\section{Clients' Assessment of Type, Cost and Quality of Services Received}

Most women were not able to indicate (69\%) the type of abortion procedure they had undergone.

The rest mentioned electrical vacuum aspiration (21\%), D\&C (6\%) and manual vacuum aspiration (5\%). The cost of services was assessed in terms of the average time it took the client to reach the facility, the amount of time she had to wait to receive services and the amount charged for the services received. As indicated in Table 11, the average amount of time taken to reach the facility was 43 minutes, and ranged from 0-120 minutes. Clients accessing public facilities took about 11 minutes longer than those visiting private facilities to reach their destinations but this difference was not significant at the $95 \%$ confidence level. Waiting times also ranged from 0 to 300 minutes but on average clients had to wait for less than an hour (46 minutes) before seeing a provider. There were no differences in waiting times between public and private sector clients. 
Table 10: Quality and cost of care received by type of sector

\begin{tabular}{|c|c|c|c|}
\hline Indicator & $\begin{array}{l}\text { Public } \\
(\mathrm{N}=70)\end{array}$ & $\begin{array}{l}\text { Private } \\
(\mathrm{N}=76)\end{array}$ & $\begin{array}{l}\text { Total } \\
(\mathrm{N}=146)\end{array}$ \\
\hline $\begin{array}{l}\text { Cost of care } \\
\text { Average travel time (minutes)* } \\
\text { Average waiting time (minutes) } \\
\text { Average cost of a one-way travel to the clinic }(\phi)^{* * *} \\
\text { Average cost of care }(\phi)^{* * *}\end{array}$ & $\begin{array}{l}49.6 \\
46.4 \\
23,046 \\
369,921\end{array}$ & $\begin{array}{l}38.8 \\
45.7 \\
4,851 \\
270,609 \\
\end{array}$ & $\begin{array}{l}43.4 \\
46.0 \\
12,670 \\
318,007\end{array}$ \\
\hline Quality of care & $\%$ & $\%$ & $\%$ \\
\hline $\begin{array}{l}\text { Clarity of explanation about the procedure } * * * \\
\text { No explanation given } \\
\text { Provider explained clearly } \\
\text { Provider explained somewhat clearly } \\
\text { Explanation not clear at all }\end{array}$ & $\begin{array}{l}21.4 \\
52.9 \\
24.3 \\
1.4\end{array}$ & $\begin{array}{l}25.0 \\
73.7 \\
0.0 \\
1.3\end{array}$ & $\begin{array}{l}23.3 \\
63.7 \\
11.6 \\
1.4\end{array}$ \\
\hline $\begin{array}{l}\text { Reception/handling by } \text { provider*** } \\
\text { Treated very well } \\
\text { Treated so-so } \\
\text { Treated poorly }\end{array}$ & $\begin{array}{l}62.9 \\
0.0 \\
37.1\end{array}$ & $\begin{array}{l}98.7 \\
0.0 \\
1.3\end{array}$ & $\begin{array}{l}81.5 \\
0.0 \\
18.5\end{array}$ \\
\hline$\%$ of clients educated on family planning $* * *$ & 45.7 & 97.4 & 72.6 \\
\hline
\end{tabular}

The average cost of a one-way trip to the facility was $\phi 12,670$ and was significantly much lower for clients accessing care at private facilities than for those seeking care at public health facilities (refer to Table 10). In terms of actual services received, the cost per service ranged from $\phi 0.0$ to $\notin 750,000$, with clients paying an average of $\phi 318,007$. The mean cost per procedure was significantly higher in the public health facilities $(\phi 369,921)$ than in the private health facilities $(ф 270,609)$. It must be noted that outliers have been excluded from the analysis on cost of care. One reason for the higher costs in the public health facilities is due to the type of management offered the client received; women presenting with complications or requiring PAC services incur greater costs.

The client's perception of the quality of care received from the provider was evaluated by examining the clarity of explanation given on the procedure which the client was expected to undergo, the type of reception received from the provider and whether or not clients were given information on how to avoid future pregnancies. While almost two-thirds (64\%) of the clients indicated that the provider had clearly explained the procedure before it was administered, about a quarter (24\%) said that they had received no explanation at all. As indicated in Table 11, 
clients seeking care at private facilities were more likely to receive clear explanations of the procedure they were going to receive than those accessing care in public facilities. In terms of the quality of treatment or handling received from the provider, about 4 out of 5 clients $(82 \%)$ said they had been treated very well. Almost all clients in the private facilities (99\%) felt that they had been treated very well by the provider compared to 63 percent of those attending public health facilities. These client reports are consistent with data reported earlier from observations of consultations.

Future unwanted pregnancies can be avoided if abortion seeking clients are provided information on how to avoid or prevent such pregnancies. The data in Table 11 suggest that about 37 percent of clients are not counseled on avoiding unwanted pregnancies. Clients seeking care in the public health institutions (54\%) tend to get no information unlike clients visiting private health institutions (3\%). A little over half the women (56\%) who had received information about how to avoid a pregnancy accepted a method. The figure varied significant from 19 percent in public health institutions to 72 percent in private health institutions $(\mathrm{P}$-value=0.000). Methods accepted by clients included the pill (24\%), injectable (17\%), the IUD (15\%), Norplant (14\%) and condoms $(2 \%)$.

Clients were further asked about the degree of their satisfaction with the services they had received at the facilities they had visited. Table 12 shows that 94 percent of clients were satisfied or very satisfied with the services they received.

Table 11: Client satisfaction with services received by type of sector

\begin{tabular}{|c|c|c|c|}
\hline \multirow[t]{2}{*}{ Indicator } & \multicolumn{3}{|c|}{$\%$ of clients } \\
\hline & $\begin{array}{l}\text { Public } \\
(\mathrm{N}=70)\end{array}$ & $\begin{array}{l}\text { Private } \\
(\mathrm{N}=76)\end{array}$ & $\begin{array}{l}\text { Total } \\
(\mathrm{N}=146)\end{array}$ \\
\hline \multicolumn{4}{|l|}{ Degree of satisfaction with services received $* * *$} \\
\hline Very satisfied & 45.7 & 94.7 & 71.2 \\
\hline Satisfied & 44.3 & 2.6 & 22.6 \\
\hline Neutral & 5.7 & 2.6 & 4.1 \\
\hline Unsatisfied & 2.9 & 0.0 & 1.4 \\
\hline Very unsatisfied & 1.4 & 0.0 & 0.7 \\
\hline Clients who would recommend facility to a friend* & 90.0 & 97.4 & 93.8 \\
\hline$* * * \mathrm{P}<0.001$ & & $\mathrm{P}<0.01$ & \\
\hline
\end{tabular}

Source: Client Exit Interviews

The degree of satisfaction was significantly much better among clients seeking care at private facilities. Recognizing that women seeking abortions may just be happy to get any service and have a problem taken care might report satisfaction, the assessment collected data on whether 
they would recommend the facility to others. Almost 94 percent of the clients said they would recommend the facility they had visited to a friend, suggesting that clients were genuinely satisfied with the services they received from the facilities. The distribution by type of sector indicates that clients accessing care at private facilities were more likely to recommend the facility to someone else.

\section{SUMMARY AND CONCLUSIONS}

This assessment found that there is a demand for safe abortion services. Safe abortion services are available in both the public and private facilities and women seek services from facilities in both sectors. At public sector facilities, women seeking care often present with complications such as heavy bleeding; while those who visit private sector clinics seek elective abortion services. Private sector facilities provide abortion services for gestations less than 12 weeks and refer women beyond the gestational age limit and those with complications to public sector facilities.

Many women who seek abortion care are young. Most of the unwanted pregnancies arise from non-use of contraception. Women who have an unwanted pregnancy go through many various intermediaries and processes (including drinking of herbal concoctions, ingestion of various types of medications, consultation of chemical sellers and pharmacists) before reaching the health facility.

Although abortion services are available, the assessment identified many quality gaps. In particular, significant gaps were noted in the quality of client-provider consultations. Providers did not ask women relevant questions pertaining to their situation and did not provide correct or adequate information. Women need to be given sufficient information so that they can avoid unwanted pregnancies and repeat abortions in the future. In particular, information on contraceptive options and family planning services needs to be provided. The integration of family planning and abortion services within the public sector needs to be strengthened.

The following are programmatic options to strengthen abortion services and contribute to reductions in maternal mortality. 
1. Improve the quality of existing services so that unwanted pregnancies and repeat abortions can be avoided. In particular, provide comprehensive information and family planning services to those sexually active; youth who may not be knowledgeable about sexuality and reproduction may have additional informational needs.

2. Improve access to services by training more providers and offering comprehensive abortion care services at more facilities in the public and private sectors. More providers can be trained in medication abortion as this method can be provided safely and effectively at facilities.

3. Educate community members on the location of existing facilities that provide safe and confidential services so that utilization at health facilities increases. Undertake community outreach activities to destigmatize legal abortion and use of contraceptives. 


\section{REFERENCES}

Ahiadeke, Clement. 2001. "Incidence of induced abortion in Southern Ghana," International Family Planning Perspectives, 27(2): 96-101 \& 108.

Awusabo-Asare, Kofi, Albert M. Abane, and Akwasi Kumi-Kyereme. 2004. "Adolescent Sexual and Reproductive Health in Ghana: A Synthesis of Research Evidence", Occasional Report, No. 13. New York: The Alan Guttmacher Institute.

Baiden, F, K. Amponsa-Achiano, A.R. Oduro, T.A. Mensah, R. Baiden, and A. Hodgson. 2006. "Unmet need for essential obstetric services in a rural district in northern Ghana: complications of unsafe abortions remain a major cause of mortality," Public Health: 120(5): 421-426.

Ghana Health Service, 2006. Prevention and Management of Unsafe Abortion: Comprehensive Abortion Care Services. Standards and Protocols, June 2006, page 1

Ministry of Health, 2007. The Ghana Health Sector Annual Programme of Work, pages 13-14

Odoi-Agyarko, Henrietta. 2003. Profile of Reproductive Health Situation in Ghana. Document prepared for WHO.

ORC Macro. 2005. Trends in Demographic, Family Planning, and Health Indicators in Ghana, 1960-2003: Trend Analysis of Demographic and Health Surveys Data. Calverton, Maryland, USA: ORC Macro.

Otsea, Karen. 2004. Lives Worth Saving: Abortion Care in sub-Saharan Africa since ICPD. A progress report. Chapel Hill: Ipas.

Srofenyoh, E.K. and A.T. Lassey. 2003. "Abortion care in a teaching hospital in Ghana," International Journal of Gynecology and Obstetrics, International Journal of Gynecology and Obstetrics 82(1): 77-78.

Quality Health Partners and Ghana Health Service. 2005. Facility baseline assessment of regional hospitals and facilities in 28 target districts in seven regions of Ghana" (Accra, Ghana: Quality Health Partners).

World Health Organization. 2006. "World Health Statistics, 2006”, Geneva, Switzerland, p 24. 